\title{
Associação entre condições de trabalho e os Indicadores de Prazer e Sofrimento no cotidiano de trabalho de Agentes Comunitários de Saúde
}

Amanda Ferreira de Araújo, Rosangela Maria Greco

\section{RESUMO}

Motivação: 0 objetivo deste estudo foi analisar e discutir condições de trabalho do Agente Comunitário de Saúde (ACS) de um município da Zona da Mata mineira e sua relação com as vivências de prazer e sofrimento no trabalho.

Métodos: É um transversal, quantitativo, realizado com 400 ACS. Utilizou-se dois questionários auto-preenchíveis e uma escala do Inventário sobre Trabalho e Risco de Adoecimento (ITRA). A população estudada foi composta por mulheres $(91,2 \%)$, com idade média de 46 anos, casadas ou em união estável $(57,5 \%)$ e com ensino médio completo $(76,4 \%)$.

Resultados: Nos resultados, a Escala de Indicadores de Prazer e Sofrimento no Trabalho (EIPST) apresentou os domínios de prazer classificados como satisfatórios. $O$ domínio Esgotamento Profissional apresentou a avaliação mais negativa, com todos os itens avaliados como críticos.

Conclusões: Verificamos que a maioria dos itens pesquisados no questionário sobre condições físico-estruturais mostrou associação estatisticamente significativa com a EIPST.

Palavras-chave: Condições de trabalho; Saúde do trabalhador; Agente Comunitário de Saúde; Prazer; Sofrimento mental.
Revista da Rede APS 2019

Publicada em: 17/12/2019

DOI:10.14295/aps.v1i3.46

Amanda Ferreira de Araújo (Universidade Federal de Juiz de Fora, Juiz de Fora, Minas Gerais, Brasil);

Rosangela Maria Greco

(Universidade Federal de Juiz de Fora, Juiz de Fora, Minas Gerais, Brasil);

Correspondência para:

Amanda Ferreira de Araújo afaufjf@hotmail.com 


\section{INTRODUÇÃO}

O Agente Comunitário de Saúde (ACS) é fundamental no contexto da Atenção Primária à Saúde (APS) no Brasil e encontra-se envolvido em uma variedade de ambientes de trabalho e peculiaridades que justificam a realização de pesquisas referentes à saúde e condições de trabalho destes profissionais.

O trabalho vai além de um meio de sustento, sendo, acima de tudo, uma forma de inserção social onde fenômenos físicos e psíquicos atuam e se entrelaçam. Por um lado, pode se constituir em fator de deterioração, envelhecimento precoce e doenças graves, por outro, pode ser fator de equilíbrio e desenvolvimento (DEJOURS; DÉSSORS; DÉSRIAUX, 1993).

Nesse contexto teve início os estudos sobre a Psicopatologia do Trabalho, hoje denominada Psicodinâmica do Trabalho, que busca identificar as formas de organização do trabalho e sua ligação com o prazer e o sofrimento que se traduz em adoecimento.

A Psicodinâmica do Trabalho compreende os mecanismos utilizados pelo trabalhador para manter o equilíbrio mental frente às condições de trabalho adversas, e as estratégias individuais e coletivas na transformação do sofrimento em prazer (DEJOURS, 1994). Ela não se limita ao impacto negativo deste, mas investiga também o cenário no qual a relação laboral é favorável à saúde (DEJOURS, 2007).

Os ACS são fundamentais na Estratégia Saúde da Família. São eles o elo entre a comunidade e profissionais e, apesar disso, estão expostos a condições de trabalho que podem gerar adoecimento e sofrimento (LOTTA, 2012; PEREIRA et al., 2014; CARNEIRO, MARTINS, 2015).

Ribeiro (2012) afirma que a saúde dos ACS está num caminho que, embora penoso, vislumbra mudanças em sua conjuntura levando em conta fatores como criatividade, ações inovadoras e organização do trabalho.
Assim sendo, o objetivo deste estudo foi analisar e discutir as condições de trabalho dos ACS de um município da Zona da Mata mineira e sua relação com as vivências de prazer e sofrimento no trabalho.

\section{MÉTOdOS}

Trata-se de pesquisa quantitativa, exploratória de corte transversal que integra uma investigação mais ampla denominada Trabalhadores da Atenção Primária à Saúde: condições de trabalho e de vida. A população foi constituída pelos 500 ACS que estavam exercendo suas atividades laborais nas UBS, tendo sido excluídos aqueles que não foram encontrados após 3 (três) tentativas de contato, totalizando 400 (80\%) ACS.

Foram selecionadas como variáveis independentes as características sociodemográficas: idade, cor da pele, situação conjugal, sexo, escolaridade, religião, filhos e renda familiar em saláriosmínimos, e como variáveis dependentes a realização profissional, a liberdade de expressão, o esgotamento profissional e a falta de reconhecimento avaliados por meio da Escala EIPST, que faz parte do ITRA.

Os dados foram tratados no programa Statistical Package for the Social Sciences (SPSS). A análise foi realizada por meio de estatística descritiva e bivariada dos dados socioeconômicos, demográficos, características e condições do trabalho.

Para o cálculo dos domínios da EIPST considerou-se como resultados, para as vivências de prazer, ponderando um desvio-padrão para cada média: acima de 4,0 (avaliação mais positiva, satisfatória), entre 3,9 e 2,1 (avaliação moderada, crítico), abaixo de 2,0 (avaliação mais negativa, grave). $E$ para os resultados das vivências de sofrimento, também considerando desvio-padrão para cada média: acima de 4,0 (avaliação mais negativa, grave), entre 3,9 e 2,1 (avaliação moderada, crítico), abaixo de 2,0 (avaliação menos negativa, satisfatório) (MENDES, 2007). 
Considerou-se associação significativa entre as variáveis estudadas quando $p$-valor $\leq 0,05$ de acordo com o teste estatístico qui-quadrado.

O projeto foi aprovado pelo Comitê de Ética e Pesquisa da Universidade Federal de Juiz de Fora sob CAAE no 40343414.0.0000.5147.

\section{Resultados}

Os resultados demonstram que $59,4 \%$ dos ACS têm idade entre 40 e 59 anos, a idade mínima foi de 25 e a máxima de 74 anos, a média de idade 46 anos, e um desvio padrão de 10,22. A maioria dos pesquisados se autodeclara de cor preta ou parda $(52,1 \%)$, são casados ou vivem em união estável (57,5\%), têm filhos $(77,5 \%)$ e se intitulam católicos (58,8\%). Chama atenção a predominância de ACS do sexo feminino (364), totalizando 91,2\% da população pesquisada. Com relação à escolaridade, $80,3 \%$ dos ACS possuem nível fundamental ou médio. Um total de $79,4 \%$ dos participantes recebe renda média mensal de até 2,5 salários-mínimos.

Os indicadores de prazer, um dos domínios do EIPST, apresentaram associação com três das variáveis socioeconômicas: idade $(p=0,001)$, escolaridade $(p=0,000)$ e filhos $(p=0,040)$. Havendo diferenças entre as médias das categorias destas variáveis, utilizou-se o teste de Tukey para verificarmos essas diferenças. $O$ teste indicou que o item "60 anos ou mais" se difere estatisticamente dos demais, portanto, pode-se afirmar que com 60 anos ou mais experimenta-se maior sensação de prazer do que em outras idades, muito embora todos os itens tenham obtido avaliação satisfatória.

O mesmo acontece com o nível de escolaridade e a presença de filhos. Os ACS que possuem filhos e um menor nível de escolaridade demonstraram um leve aumento na média da vivência de prazer, sendo mais satisfatória do que aqueles que não possuem filhos e possuem uma escolaridade superior.
Apenas a variável "sexo" se associou aos indicadores de sofrimento. Ambos os gêneros mostraram sofrimento crítico em suas vivências no trabalho, porém, as mulheres tiveram uma média mais elevada.

\section{DIscussÃo}

Com relação à idade, os ACS são adultos jovens, no auge da capacidade produtiva da vida. A perspectiva de mudança na previdência social, com a ampliação da idade e tempo de trabalho para aposentadoria, mostra a necessidade de uma atenção ainda maior para condições de trabalho adequadas para que estes trabalhadores possam permanecer saudáveis durante toda a sua vida laboral. Dados semelhantes foram achados no relatório final da Universidade Federal da Bahia (2015) e nos estudos de Barbosa (2014), Lino (2013) e Lopes et al. (2012).

Anchieta et al. (2011) refere que os trabalhadores com menos idade e que estão iniciando a vida profissional têm muitas expectativas em relação ao trabalho e, quando estas expectativas não são atendidas, pode ser um fator gerador de sofrimento e adoecimento se não forem criadas estratégias defensivas.

Quanto à cor da pele, $52,1 \%$ se autodeclararam pretos ou pardos, demonstrando a diminuição da rejeição com relação às raças historicamente mais susceptíveis ao preconceito racial (UNIVERSIDADE FEDERAL DA BAHIA, 2015; OSÓRIO, 2013).

Em relação ao gênero, a predominância do sexo feminino também foi encontrada nos estudos de Barbosa (2014), Lino (2013), Mascarenhas, Prado e Fernandes (2013), Lopes et al. (2012) e Marzari, Jungles e Selli (2011), além de relatório de estudo realizado pela Universidade Federal da Bahia (2015), com ACS de todo território brasileiro. É importante destacar o processo de feminização das profissões e ocupações da área da saúde. Trata-se de um processo de construção histórica e cultural, em que a sociedade atribui à mulher o papel de cuidadora. Esta concepção expressa uma divisão social e sexual do trabalho, na qual a sociedade 
delimita os campos em que a mulher pode operar ou encontra-se mais "apta" a trabalhar (LINO et al., 2012; MACHADO, 2006). Neste sentido, a predominância de mulheres desempenhando a função de ACS está fortemente relacionada ao papel de cuidadora historicamente atribuído à mulher.

A EIPST (indicadores de sofrimento) mostrou associação significativa apenas com a variável "sexo", demonstrando que as mulheres tendem a ter uma vivência mais expressiva de sofrimento que os homens. Segundo Mascarenhas, Prado e Fernandes (2013), isso acontece devido à dupla jornada de trabalho da mulher, tendo que trabalhar e cuidar da casa e dos filhos, o que acaba gerando uma série de conflitos uma vez que o excesso de atividades limita o tempo de estar com a família e de desenvolver atividades que a realize pessoalmente, prejudicando seu desempenho como mãe, esposa, filha e amiga.

A maioria dos pesquisados eram casados ou viviam em união estável (57,5\%), possuíam filhos (77,5\%) e professavam a religião católica $(58,8 \%)$. Vale ressaltar a importância da religiosidade no contexto histórico-social, influenciando também na concepção de saúde dos indivíduos. Tal fato permite ao ACS uma maior interação com a comunidade, em que a religião serve de elo e o ACS, católico em sua maioria, deverá respeitar os demais credos (UNIVERSIDADE FEDERAL DA BAHIA, 2015; LINO et al., 2012; URSINE; TRELHA; NUNES, 2010).

Quanto à família, a maioria dos ACS já havia passado pela experiência da maternidade ou paternidade, o que pode contribuir para que eles tragam vivências pessoais sobre saúde infantil oriundas dos cuidados com seus filhos. Se por um lado isso gera um conhecimento prévio que pode facilitar e dar mais segurança no trabalho de orientação junto às famílias, por outro os ACS não devem confundir as experiências pessoais com as profissionais, pois o que foi adequado para um caso pode não o ser para o outro. Por isso a importância da educação permanente e da supervisão de seu trabalho pela equipe de saúde (FERRAZ; AERTZ, 2005).
Os ACS que possuem filhos e um menor nível de escolaridade demonstraram um leve aumento na média da vivência de prazer, sendo mais satisfatória do que aqueles que não possuem filhos e possuem uma escolaridade superior. Outros estudos apresentaram resultados semelhantes (GLANZNER, 2014; MAISSIAT, 2013) para "idade" e "escolaridade".

Podemos inferir que os ACS com mais escolaridade possuem mais segurança, como decorrência de maior conhecimento, o que auxilia também na capacidade de iniciativa e tomada de decisão, sendo que o mesmo pode-se inferir em relação aos filhos, o que confere uma experiência que muitas vezes é significativa.

Com relação à escolaridade, $76,4 \%$ dos profissionais pesquisados possuem o ensino médio e $19,7 \%$ o ensino superior, sendo que apenas 3,9\% cursaram até 0 ensino fundamental. Isso demonstra que os ACS possuem formação educacional superior à que é preconizada pela Lei 11.350/2006, que regulamentava a profissão e apontava como exigência para exercê-la apenas o ensino fundamental (BRASIL, 2006). Este estudo não nos revela o motivo para tal fato, porém, pode-se supor que isso se deve, em parte, à dedicação desses profissionais ao trabalho fazendo-os sentirem necessidade de estarem mais bem preparados para exercerem o seu ofício. Por outro lado, a carreira de ACS tem se mostrado como uma possibilidade para aqueles que, muitas vezes, não conseguem se inserir no mercado de trabalho.

Em relação à classificação econômica, a maioria dos trabalhadores referiu receber entre dois e quatro salários mínimos (51,4\%). Segundo informações de pesquisa realizada pelo Instituto Brasileiro de Geografia e Estatística(BRASIL, 2018) de abrangência nacional, o rendimento médio real de todos os trabalhos foi 2,28 salários mínimos. Fazendo uma comparação com os trabalhadores de nosso estudo, podemos dizer que a renda média mensal destes está dentro da média geral de renda da população do país, porém estamos nos referindo à média salarial familiar e não ao salário da categoria, o qual, de fato, é baixo 
perante a realidade em que este trabalhador se insere e aos riscos aos quais está sujeito (SANTANA et al., 2009).

Dentre as limitações deste estudo, temos que considerar o viés de prevalência, visto que não foi possível avaliar os trabalhadores de licença/afastamento, e os que foram demitidos/exonerados, considerando para este fato o efeito do trabalhador sadio (MAISSIAT, 2013; LINO, 2013). Há, também, um viés de causalidade, característica dos estudos transversais (LINO, 2013), já que não é possível observar as vivências de prazer e sofrimento dos ACS anteriores ao estudo e não será possível prever tais características posteriormente.

Segundo Mendes (2007), o ITRA, quando utilizado para estudos sobre saúde e trabalho, apresenta um cenário, capaz de capturar a realidade representada na sua versão mais exposta e divulgada pela maioria dos trabalhadores de um determinado grupo profissional, ganhando dados gerais (objetivos, quantitativos), mas perdendo as observações específicas (subjetivas, qualitativas). Para isso, faz-se necessário, posteriormente, realizar um estudo qualitativo abordando a mesma temática.

\section{CONSIDERAÇõES FINAIS}

O presente estudo buscou analisar e discutir as condições de trabalho dos ACS de Juiz de Fora, município de grande porte do estado de Minas Gerais. Para tanto, traçamos um perfil sociodemográfico e de condições físico-estruturais de trabalho da população estudada e utilizamos o ITRA como instrumento complementar.

A alta predominância do gênero feminino reflete a feminização dos profissionais de saúde, demonstrando a divisão sexual do trabalho. Esta perspectiva de gênero nos leva a uma inquietação a respeito do modo como a predominância do sexo feminino na pesquisa pode ter influenciado o resultado obtido. Trata-se de um fator importante a ser investigado posteriormente em outro estudo.
Constitui ponto positivo desta investigação o fato dos ACS possuírem estabilidade no emprego, o que contribui com a possibilidade de monitoramento do trabalhador em longo prazo, além de ser um fator de valorização do profissional, diminuindo a precarização do trabalho e aumentando as vivências de prazer desses funcionários.

Dentre as limitações deste estudo podemos citar o fato desta pesquisa ser delineada por um estudo transversal que tem a finalidade de fazer um recorte de uma determinada realidade, para que a mesma possa ser analisada, explorada. Devido a estas características do estudo transversal não nos é possível acompanhar as condições de trabalho desses trabalhadores ao longo dos anos ou mesmo avaliar condições anteriores. Além disso, o instrumento de coleta de dados utilizado foi o questionário autoprenchível, que apesar de ser adequado a este tipo de delineamento, não expressa todas as questões que envolvem o ser humano.

Como contribuições da saúde coletiva para a melhoria das condições de trabalho desses profissionais, sugerimos uma readequação dos postos de trabalho, além de fornecimento de informações a respeito dos riscos ocupacionais a que esses trabalhadores estão submetidos para que estes tomem consciência dos danos que podem ser ocasionados ao longo do tempo e, consequentemente, realizem a prevenção adequada. Com relação ao poder público e gestores, sugerimos maiores investimentos na força de trabalho para que possa se adequar ao que está preconizado na Política Nacional de Saúde do Trabalhador e da Trabalhadora (2012).

\section{REFERÊNCIAS BIBLIOGRÁFICAS}

ANCHIETA, VCC; GALINKIN, AL; MENDES, A MB; NEIVA, ER. Trabalho e Riscos de Adoecimento: Um Estudo entre Policiais Civis. Psicologia: Teoria e Pesquisa. Vol. 27, n. 2, p. 199-208, abr/jun 2011.

BARBOSA, AM. Análise das Condições de Saúde de Agentes Comunitários de Saúde: relação com 
Vol. 1, n. 3, p. 173/180 | Setembro/Dezembro- 2019

ISSN 2596-3317-DOI 10.14295/aps.v1i3.46

Araújo, A. F.; Greco, R. M.

perfil nutricional e capacidade para o trabalho. $55 f$. Monografia (graduação) - Universidade Federal da Paraíba. Centro de Ciências da Saúde. Departamento de Nutrição. João Pessoa - PB, 2014.

BRASIL. Lei no 13.595, de 05 de janeiro de 2018. Altera a Lei no 11.350 , de 5 de outubro de 2006 , para dispor sobre a reformulação das atribuições, a jornada e as condições de trabalho, o grau de formação profissional, os cursos de formação técnica e continuada e a indenização de transporte dos profissionais Agentes Comunitários de Saúde e Agentes de Combate às Endemias. Diário Oficial da União, 09 de janeiro de 2018.

BRASIL. Portaria n. -1.823 , de 23 de agosto de 2012. Institui a Política Nacional de Saúde do Trabalhador e da Trabalhadora. Diário Oficial da União, 24 de agosto de 2012.

BRASIL. Lei no 11.350, de 05 de outubro de 2006 . Regulamenta o § 50 do art 198 da Constituição, dispõe sobre o aproveitamento de pessoal amparado pelo parágrafo único do art 20 da Emenda Constitucional no 51, de 14 de fevereiro de 2006, e dá outras providências. Diário Oficial da União, 06 de outubro de 2006.

DEJOURS, C; DÉSSORS, D; DÉSRIAUX, F. Por um trabalho, fator de equilíbrio. Revista de Administração de Empresas, RAE, [s.l.], v.33, n.3, p. 98-104, 1993.

DEJOURS, C. Psicodinâmica do Trabalho: contribuições da Escola Dejouriana à análise da relação prazer, sofrimento e trabalho. São Paulo: Atlas, 1994

DEJOURS, CA. Psicodinâmica do trabalho na pósmodernidade. In: MENDES, A M; LIMA, SCC; FACAS, EP. Diálogos em psicodinâmica do trabalho. Brasília: Paralelo; 2007.

CARNEIRO, CCG; MARTINS, MIC. Novos modelos de gestão do trabalho no setor público de saúde e o trabalho do agente comunitário de saúde. Trab. Educ. Saúde, Rio de Janeiro, v. 13, n. 1, p. 45-66, abr. 2015
FERRAZ, L.; AERTZ, DRGC. O cotidiano de trabalho do agente comunitário de saúde no PSF em Porto Alegre. Cien Saúde Colet, v. 10, n. 2, p. 347-355, 2005.

GLANZNER, $\mathrm{CH}$. O descompasso entre o trabalho real e o prescrito: prazer e sofrimento dos profissionais das equipes de saúde da família no Grupo Hospitalar Conceição. 217 f. Tese (doutorado) - Universidade Federal do Rio Grande do Sul, Escola de Enfermagem, Programa de PósGraduação em Enfermagem. Porto Alegre - RS, 2014.

LINO, DCSF. Saúde Mental e Condições de Trabalho entre Agentes Comunitários de Saúde. 111f. Dissertação (mestrado) - Universidade Estadual do Sudoeste da Bahia. Programa de PósGraduação em Enfermagem e Saúde. Jequié - BA, 2013.

LINO, MM; LANZONI, GMM; ALBUQUERQUE, GL; SCHVEITZER, MC. Perfil socioeconômico, demográfico e de trabalho dos Agentes Comunitários de Saúde. CogitareEnferm. V. 17, n. 1, p. 57-64, 2012.

LOPES, DMQ; BECK, CLC.; PRESTES, FC; WEILLER, TH; COLOMÉ, JS; SILVA, GM. Agentes Comunitários de Saúde e as vivências de prazer - sofrimento no trabalho: um estudo qualitativo. Rev. Esc. Enferm. USP, São Paulo, v. 46, n. 3, p. 633-40, 2012.

LOTTA, GS. Saberes Locais, Mediação e Cidadania: o caso dos agentes comunitários de saúde. Saúde Soc., São Paulo, v. 21, supl. 1, p. 210-222, 2012.

MACHADO, JMH; SANTANA, V. 10 Inventário de Saúde do Trabalhador, 2009: Avaliação da Rede Nacional de Atenção Integral em Saúde do Trabalhador. MACHADO, MH. Trabalhadores da Saúde e sua Trajetória na Reforma Sanitária. In: BRASIL. Ministério da Saúde. Secretaria de Gestão do Trabalho e da Educação na Saúde. Cadernos RH Saúde. v. 3, n. 1. Brasília: Ministério da Saúde, p. 13-28. mar. 2006

MAISSIAT, GS. Prazer e Sofrimento de Trabalhadores da Atenção Básica. Saúde à Luz da Teoria da Psicodinâmica do Trabalho. 114f. 
Vol. 1, n. 3, p. 173/180 | Setembro/Dezembro- 2019

ISSN 2596-3317-DOI 10.14295/aps.v1i3.46

Araújo, A. F.; Greco, R. M.

Dissertação (Mestrado em Enfermagem) Programa de Pós-Graduação em Enfermagem Escola de Enfermagem. Universidade Federal do Rio Grande do Sul. Porto Alegre, 2013.

MARZARI, CK; JUNGES, JR; SELLI, L. Agentes Comunitários de Saúde: perfil e formação. Cien Saúde Colet, v. 16 (Supl. 1), p. 873-880, 2011.

MASCARENHAS, CHM; PRADO, FO; FERNANDES, $\mathrm{MH}$. Fatores associados à qualidade de vida de Agentes Comunitários de Saúde. Cien Saúde Colet, v. 18, n. 5, p. 1375-1386, 2013.

MENDES, AM. Psicodinâmica do trabalho: teoria, método e pesquisa. São Paulo: Casa do Psicólogo, 2007.

OSORIO, RG. A classificação de cor ou raça do IBGE revisada. In: Ministério do Planejamento, Orçamento e Gestão. Instituto Brasileiro de Geografia e Estatística. PETRUCCELLI, JL; SABOIA, AL. (orgs). Características Étnico-raciais da População: Classificações e identidades. Estudos e Análises. Informação Demográfica e Socioeconômica n. 2, IBGE, p. 83-99, 2013.

PEREIRA, IVS; ROCHA, MJL; SILVA, VM; CALDEIRA, AP. Morbidade autorreferida por trabalhadores das Equipes de Saúde da Família. Cien Saúde Colet, Rio de Janeiro , v. 19, n. 2, p. 461-468, feb. 2014.

RIBEIRO, SFR. Agente Comunitário de Saúde: Dificuldades no Processo de Construção Histórica da Profissão e Adoecimento. Rev. Laborativa, v. 1, n. 1, p. 17-35, out./2012.

SANTANA, JCB; VASCONCELOS, AL; MARTINS, CV; BARROS, JV; SOARES, JM; DUTRA, BS. Agente Comunitário de Saúde: Percepções na Estratégia Saúde da Família. CogitareEnferm. V. 14, n. 4, p. 645-52, 2009.

UNIVERSIDADE FEDERAL DA BAHIA. Instituto de Saúde Coletiva. Relatório final: Avaliação do perfil dos Agentes Comunitários de Saúde no processo de consolidação da Atenção Primária à Saúde no Brasil. Salvador: UFBA, 2015.

URSINE, BL; TRELHA, CS; NUNES, EFPA. O Agente Comunitário de Saúde na Estratégia Saúde da
Família: uma investigação das condições de trabalho e da qualidade de vida. Rev. Bras. Saúde Ocup. São Paulo, v. 35, n.122, p. 327-339, 2010.

cc) 


\section{ABSTRACT}

Background: The purpose of this study was to analyze and discuss the working conditions of the Community Health Agents (ACS) from a municipality of Minas Gerais and its relationship with the experiences of pleasure and suffering at work.

Methods: A cross-sectional, quantitative study was carried out with 400 ACS. Self-fulfilling surveys and scales of the Work Inventory and Risk of Falling ill (ITRA) were used as instruments. The population studied was female (91.2\%), around 46 years old in average, married or in a stable relationship (57.5\%) with a high school degree of education (76.4\%).

Results: In the results, the Pleasure and Suffering at Work Indicators scale (EIPST) presented the domains that evaluate pleasure classified as satisfactory. The Professional Exhaustion domain presented the most negative evaluation, with all the items evaluated as critical.

Conclusions: We check the most of searched items in the physical and structural questionnaire showed association statistically significant with EIPST.

Keywords: Working conditions; Worker's health; Community Health Agent; Pleasure; Suffering mental. 